\title{
To sleep or not to sleep...
}

Wind turbines are seen by many as the lowest cost green energy sources, apart from hydro-power turbines. However, their intermittent nature currently plays havoc with the stability of electricity networks that rely too heavily upon them. Battery storage is seen as a possible means of smoothing out the power fluctuations associated with a particular wind farm, but it is currently too expensive to be in common use. Where mountainous terrains exist, there is a possibility of using pumped hydro storage to even out the fluctuations with an expected efficiency of 80

There are a number of aspects of wind farm noise that result in it being highly annoying and sleep disturbing to a percentage of the population. The exact percentage varies between countries and between rural and suburban areas and is a function of the level and character of background noise that existed prior to construction of the wind farm. The aspects of wind farm noise that are potentially disturbing to sleep include low frequency dominance when experienced indoors, the presence of periodic infrasound (having a considerably different character from normal environmental infrasound), tonality, and periodic amplitude modulation (varying usually at the blade rotational speed) as well as random amplitude variation. The influence of each of these aspects on a person's sleep is the subject of current research and statements, made in the media and by some social science professionals, about disturbances from wind farm noise being a detrimental effect are uninformed at best and malicious at worst. It is easy to dismiss people's complaints and suggest that they must be suffering from the detrimental effect, as many people who visit a wind farm during daytime barely notice the associated noise. Nevertheless, there are numerous documented cases in many countries where people have to leave their homes, as they find living near a wind farm to be unbearable because of the noise. Many others cannot afford to leave but suffer a significantly degraded quality of life and a significant loss of sleep, not to mention the stress arising from being regularly annoyed by the noise.

There are four main reasons why wind farm noise can be more apparent at night time when people are trying to sleep. First, the walls and roof of a residence act as low pass filters, favouring transmission of low-frequency noise resulting in a more annoying, low-frequency dominated spectrum. Second, background noise levels at night, especially in rural areas, are much lower at night resulting in wind farm noise being more intrusive and more dominant. Third, when some people are exposed to an annoying noise night after night, they become sensitised to it and their brain training enables them to distinguish and focus on this particular noise even when it is experienced at very low levels. Fourth, weather conditions in the early hours of the morning are often very conducive to sound propagation, resulting in higher noise levels than may be encountered during the day.

The questions we should be asking are: "How should wind farm noise regulations be decided so that an acceptable percentage of the population is protected from sleep disruption for an adequate percentage of the time? What is the acceptable percentage of people in a rural or suburban community who can have their sleep significantly disrupted? What is the tolerable number of nights in one week and in one month that the acceptable percentage of people can have their sleep disrupted?" Before these questions are answered by our politicians, more research (over significantly long time periods) needs to be conducted in order to quantify the effects of the various noise characteristics and the percentage of people affected as a function of the level associated with each characteristic. Regulations should also address the use of noise prediction models and include a requirement for uncertainty assessment as well as compliance assessment and an estimate of the percentage of time that the predicted levels are expected to be exceeded (based on annual meteorological records).

As acousticians, I believe that we have the responsibility to give the highest priority to the well-being of our community and this requires us to take seriously complaints of excessive noise by communities, even though A-weighted average noise levels may be below what we think should be annoying to most people. We should also bear in mind that the average A-weighted noise level is not necessarily an accurate measure of the annoyance or sleep disruption of a noise, especially if the noise is dominated by low-frequencies, is amplitude modulated, or is characterised by significant amplitude modulation or variation.

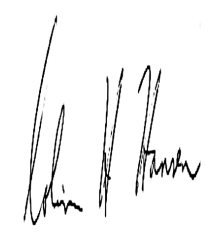

Colin H. Hansen

School of Mechanical Engineering

University of Adelaide

South Australia 